\title{
Intervista a Luigi Malerba
}

\section{Grazia Menechella}

Questa intervista ha avuto luogo a Toronto nell'ottobre del 1989. Luigi Malerba era a Toronto in quanto partecipante ad un convegno internazionale di scrittori. L'intervista è stata preceduta da una piacevole conversazione sui suoi diversi interessi ed attività. Malerba oltre ad essere un noto scrittore ha anche lavorato nel campo della pubblicità e come sceneggiatore. Anche se vive a Roma trova il tempo di dedicarsi alla campagna: ama definirsi un contadino-e un contadino all'antica, ovvero contro i pesticidi. E anche un assiduo collaboratore di Repubblica. Tra le sue opere: La scoperta dell' alfabeto, Il serpente, Salto mortale, Il protagonista, Le rose imperiali, Il pataffio, Diario di un sognatore, Il pianeta azzurro, Testa d'argento. Il suo penultimo romanzo, Il fuoco greco, è stato a lungo in testa alle classifiche dei libri più venduti in Italia. Da alcuni mesi è in libreria il suo ultimo romanzo, Le pietre volanti.

Lei è qui a Toronto per un convegno internazionale, è l'unico scrittore presente ed anche uno dei pochi autori tradotti all' estero. Che valore ha questa internazionalità e come mai, secondo Lei, l'editoria italiana non vende nel Nord America?

Le informazioni degli editori stranieri sulla produzione letteraria italiana spesso seguono percorsi tortuosi e occasionali, passano attraverso ottusi redattori oppure, e qui siamo al peggio, seguono le segnalazioni dei premi letterari, le classifiche dei libri più venduti. Come funzionano in Italia, ma non solo in Italia, i premi e le classifiche è ormai noto a tutti, ma vale la pena di ricordare per chi non segua le cronache letterarie del nostro paese, che poco più di un anno fa è entrato nella classifica dei libri più venduti compilata da una delle più "autorevoli" agenzie di sondaggi, l'ultimo romanzo di Giuseppe Patroni-Griffi prima ancora che venisse distribuito nelle librerie, con relativo scandalo. La raccolta delle Fiabe italiane di Italo Calvino è stata tradotta negli Stati Uniti, con grande successo, solo dopo trentacinque anni dalla pubblicazione in Italia, e uno scrittore come Tommaso Landolfi è praticamente sconosciuto mentre Dino Buzzati è tradotto in tutto il mondo. Se poi si prende contatto con gli italianisti che lavorano nelle università, sia qui a Toronto che negli Stati Uniti, ci si rende conto che svolgono una attività di studio e di aggiomamento di alta qualità, che i loro scritti vengono registrati da eccellenti riviste, ma che la grande editoria non comunica con loro. A differenza che in Europa, mi pare che qui il mondo accademico sia nettamente separato da quello editoriale e dai mezzi della Grande Comunicazione 
e che lavori rinserrato nelle proprie istituzioni di cui fanno parte le riviste specialistiche e l'editoria universitaria. In Europa ormai i professori universitari collaborano ai grandi quotidiani e alle riviste a larga diffusione, spesso ne costituiscono anzi la parte più attiva, efficiente e qualificata. Non so se la situazione americana sia dovuta allo "snobismo" accademico o alla inveterata diffidenza della grande editoria e della televisione nei confronti del mondo universitario. Questa mancanza di collaborazione è comunque, a mio parere, un fenomeno negativo che provoca disguidi, cattiva informazione, disordine culturale.

"Sognare di essere addormentati" e scrivere usando il linguaggio del sogno: quale è il rapporto tra sogno e scrittura?

Non vedo molta differenza tra la registrazione delle fantasie diume in forma di romanzo o racconto, e di quelle notturne in forma di diario. Il rapporto con la scrittura è lo stesso anche se nel primo caso i materiali, per assumere la forma letteraria, vengono sottoposti a una elaborazione strutturale e formale. Nel caso del sogno io ho scritto un diario (Diario di un sognatore) e naturalmente non ho elaborato letterariamente i materiali forniti dalla memoria, ma ho cercato di usare un linguaggio il più possibile neutro per non alterare la "verita" del sogno. Ho elaborato i materiali del sogno soltanto nei casi in cui ho abbandonato la forma del diario e me ne sono servito per scrivere dei racconti o delle favole (è successo più di una volta). Questi due gradi diversi del mio lavoro fanno parte della stessa necessita di tradurre tutto in scrittura, nella convinzione e illusione di fissare in questo modo le mie esperienze, di evitarne la dispersione. Anche il sogno è una esperienza e, fra le tante, quella più esposta alle ventate dispersive. Allora il sognatore che ha orrore del vuoto deposita le proprie immagini in quella immensa biblioteca o "universo della scrittura" il cui rovescio è un "universo della lettura" nel quale spera di trovare il suo confidente e depositario. Io non scrivo per me stesso come affermano narcisisticamente molti scrittori, io scrivo per essere letto. Forse la cosa difficile da accettare, e che produce atteggiamenti di disagio e di sospetto, è che il sogno resiste ai tentativi di razionalizzare tutto, di misurare e geometrizzare il mondo. I risultati di questo atteggiamento hanno sortito effetti disastrosi perché il mondo, sogno compreso, non è riducibile a formule e categorie stabili e la realtà non è geometrica.

Nel Serpente il canto è senza emissione di voce, è un canto mentale che, come il pensiero, non si sente. Come l' erotismo, il canto è una questione "di fiato e di ritmo"- e come canta bene il protagonista! Ci definisca il canto.

Il "canto mentale" raccontato nel Serpente fa parte di una Nuova Soggettività verso la quale la letteratura dovrà avviarsi necessariamente in un'epoca come la nostra in cui perfino la scienza, parlo soprattutto della fisica delle particelle, ha trovato il suo ultimo approdo conoscitivo nel soggetto. Il canto mentale è una sfida al sapere ottuso, al pensare corto, al finto realismo. 
Antropofagia nel Serpente, un radioamatore convinto di essere il proprio pene nel Protagonista, terribili "caccaiole" nel Pataffio rimandano al basso, al corporeo. Bachtin ci insegna che il "vero" riso parte dal basso e che la "vera" parodia (quella di Rabelais) nel tempo, degenerando, diventa formale. Fa ridere o no la comicità di Malerba?

Le strategie del comico non sono univoche anche se gli esempi più rumorosi e carnevaleschi partono dal basso, dal corporeo, dal rovesciamento e dalla scomposizione delle convenzioni sociali. Guido Almansi ha definito "carnalista" la comicità del Protagonista e del Pataffio, ma in altri libri ho seguito il percorso inverso, ho cercato di trascinare nel fango il "sublime", come nel canto mentale o nell'erotismo scandito sui ritmi dei capolavori della musica classica (Il serpente). Qualche volta il comico si può sviluppare addirittura nei territori ghiacciati della matematica o della geometria: la gallina disegnata sulla ipotenusa di un triangolo rettangolo equivale alla somma delle due galline disegnate sui cateti (Le galline pensierose). Ma Schopenhauer è andato più in là perché trovava comica la tangente del cerchio. La comicità di Malerba fa ridere necessariamente dal momento che si tratta di comicità, ma le mie ambizioni vanno oltre il riso, che si può ottenere anche con il solletico. Io mi ritengo soddisfatto quando, finite le parole (la lettura), il significato continua.

\section{L'ironia è un' arma, uno strumento di lavoro o altro?}

L'ironia non è soltanto una strategia o un artificio letterario, ma un atteggiamento verso il mondo. Non è un atteggiamento di sfiducia o di scetticismo come può apparire superficialmente, ma un modo di produrre quell'effetto di lontananza che è indispensabile alla narrativa se non vuole decadere nei tempi della cronaca. Senza la componente della lontananza ironica il mio romanzo Il pianeta azzurro sarebbe stato comprensibile soltanto in Italia oggi, mentre è gia uscito in Francia e sta per uscire tradotto in altri sei paesi. Šklovskij ha scritto in Zoo o lettere non d'amore che l'ironia è il mezzo più facile per vincere la difficoltà di rappresentare una cosa. È vero, ma è vero anche il contrario. Un eccesso di ironia può creare scetticismo nel lettore e ostacolare la identificazione (con il protagonista, con la storia, con l'autore) che è la molla primaria della lettura. Un bicchiere di vino facilita la comunicazione, ma l'ubriachezza la annulla. Spesso nei miei libri l'ironia è un preludio alla catastrofe, un artificio per nascondere o gettare un velo sul dramma che sta per travolgere un personaggio, insomma una forma di pudore letterario che non ho difficoltà a confessare. La serietà esibita, la coscienza estatica, la tragedia svelata, fanno parte del mondo delle certezze. Ma oggi le certezze appartengono a chi si arresta alla superficie delle cose, alle intelligenze povere. La povertà di spirito può condurre alla santita, ma letterariamente è improduttiva.

L'ironia crea una distanza col lettore ma, allo stesso tempo, piu volte questi e 
invitato a collaborare o ad essere partecipe della difficoltà del personaggio, a scrivere (nel Serpente, per esempio). Il lettore è preso in giro dall' autore?

Qualunque sia la sua temperatura, l'ironia è una finzione dentro la finzione e perciò è naturale che il lettore venga chiamato a partecipare alle difficoltà del personaggio, a sollecitarne l'azione, a rimuovere gli atteggiamenti di rinuncia. L'ironia può essere distruttiva, ma contiene i propri antidoti che, messi in funzione, neutralizzano $\mathrm{i}$ possibili effetti negativi. Il lettore non è preso in giro dall'autore, almeno nel mio caso, ma al contrario viene coinvolto e chiamato a collaborare. L'esempio massimo di questa ironia dialettica è quella socratica che mette a confronto diretto il lettore, o il discepolo, con il suo interlocutore.

Una domanda molto generale: quale è il suo ruolo sociale o etico come scrittore?

La funzione sociale è implicita nella attività dello scrittore quando non scelga (o non si rassegni) a fare da portavoce delle istituzioni, buone o cattive che siano. Non credo sia compito del romanziere (se esiste il romanzo esiste anche il romanziere per quanto brutta sia la parola) elaborare progetti realizzabili a breve scadenza, che è compito della politica, e nemmeno progetti sociali a tutto tondo, che è compito delle ideologie. Il romanzo è dunque una favola e la domanda che senso abbia scrivere una favola "nella situazione attuale", come si sente spesso ripetere, poteva essere fatta a qualsiasi scrittore in qualsiasi epoca. Kermode sostiene che è un luogo comune parlare della propria situazione storica come eccezionalmente terribile e, dunque, in un certo modo privilegiata come se fosse un punto cardinale del tempo. Vale a dire: una "situazione attuale" più o meno drammatica si è verificata in ogni tempo e in ogni luogo e gli scrittori hanno comunque inventato le loro favole più o meno condizionati dagli eventi esteriori. Eे soprattutto nelle strutture interne del racconto più che nel ricalco dei fatti, che lo scrittore è uomo del suo tempo senza che un filo diretto leghi la sua opera agli avvenimenti che si svolgono intorno a lui.

E proprio nella individuazione (ma anche nella rielaborazione o deformazione) dei segni del suo tempo che lo scrittore realizza e propone la propria utopia e con questa parola intendo i progetti, le speranze, le paure, le fantasie che sollevano l'umanità dalla contingenza immediata e le offrono una prospettiva nella quale può leggere $\mathrm{i}$ frammenti del proprio destino. I paradossi disperati di Kafka sono l'esempio piu alto di ciò che intendo io per utopia letteraria. Anche la letteratura "realistica", che affonda le sue radici e le sue ragioni negli eventi reali, acquista un senso soltanto quando i fatti assunti come materiali del racconto raggiungono una dimensione che va oltre la contingenza. I grandi scrittori realistici del passato non sopravvivono per lo spicchio di realtà che raccontano, ma per il senso che questa realtà acquista in prospettiva. Lo scrittore che aderisce alle soluzioni che gia esistono e che sono state elaborate dalla tradizione culturale fallisce in partenza anche se ha 
largo consenso di lettori. Il largo consenso può significare che lo scrittore si muove nell'area della comunicazione e non in quella della espressione dove risiede il suo impegno etico. La comunicazione si consuma senza lasciare traccia, non riguarda la letteratura né i suoi risvolti etici. Per la comunicazione sono sufficienti i giomali, la radio, la televisione, gli uffici postali e telegrafici.

Lei ha iniziato la sua carriera come sceneggiatore e ha sempre scritto per il cinema e la televisione. Lei ha scritto la riduzione cinematografica del Pianeta azzurro a proposito della quale ha affermato di aver venduto la sua ombra, forse l'anima e, sicuramente, di aver tradito il testo. Ci parli del suo rapporto con il cinema e del tradimento-adattamento.

Lavorare per il cinema e la televisione è una seconda professione che non contraddice la mia attività di scrittore. Io sostengo anzi che uno scrittore dovrebbe sempre fare anche qualcosa d'altro, non si può essere scrittori ventiquattro ore su ventiquattro. Immergersi in altre attività significa avere altre esperienze che rinnovano continuamente il magazzino delle immagini e dei sentimenti.

Malerba/Manganelli: entrambi contro il realismo e a favore di una letteratura fantastica. Entrambi etichettati postmoderni. Le sta bene questa etichetta?

In Italia Romano Luperini e Francesco Muzzioli mi hanno definito come postmoderno. Negli Stati Uniti è uscito da poco un libro di JoAnn Cannon intitolato Postmodern Italian Fiction. The Crisis of Reason in Calvino, Eco, Sciascia, Malerba. Il postmoderno è una categoria abbastanza generica, ma utile per tracciare una mappa letteraria e per impostare un discorso critico. Per quanto mi riguarda sento maggiori affinità con Calvino che con Manganelli che definirei piuttosto un manierista o un neo-barocco. Ma anche queste sono etichette generiche se non sostenute da un discorso critico.

Lei è letto anche dai piccoli; ha, infatti, sempre scritto per bambini e ragazzi. Quale è l'importanza della letteratura per bambini e come si rapporta ai piccoli lettori?

Nei miei libri per bambini e per ragazzi ho fatto ampio uso del nonsense per i più piccoli (un mio personaggio pretende di gettare la casa fuori dalla finestra, un altro inventa un recipiente grande di dentro e piccolo di fuori ecc.), del paradosso e del paralogismo per i più grandicelli (un mio personaggio scopre la velocità del buio, un altro afferma che se una automobile con quattro ruote corre a cento chilometri all'ora, una automobile con otto ruote correrà a duecento chilometri all'ora ecc.). Ma anche nei miei libri per adulti il paradosso è spesso presente (il tempo è una invenzione degli uomini per evitare che le cose accadano tutte insieme ecc.). La cosa essenziale nella letteratura per i ragazzi è prima di tutto quella di divertirli, poi di abituarli a "leggere" la realtà al di là delle convenzioni che gli vengono formite dalla 
scuola, dalla famiglia e soprattutto dai mezzi della Grande Comunicazione. Meglio un branco di caproni dialettici che un gregge di pecore dogmatiche.

University of Toronto 